\title{
Efeito do estabelecimento de metas na aprendizagem do arremesso do Basquetebol
}

\author{
Herbert Ugrinowitsch \\ Luiz E.P.B.T. Dantas
}

\author{
Escola de Educação Física e Esportes \\ Universidade de São Paulo \\ Brasil
}

\section{RESUMO}

As metas podem ser divididas em metas genéricas e metas específicas. As metas genéricas são caracterizadas por instrução do tipo "faça o melhor possível", e as metas específicas são bem definidas e, de preferência, quantitativas. Essa estratégia de estabelecer metas tem sido utilizada com atletas de alto rendimento, mas os seus efeitos na aprendizagem de habilidades esportivas ainda não estão claros. Essa questão foi investigada em uma situação real de ensino, em aulas de educação física escolar, com crianças $(n=166)$ da quinta série do ensino fundamental (11 anos), divididas em três grupos experimentais: metas genéricas (MG), metas específicas (ME) e sem metas (SM) na tarefa de arremesso do basquetebol. A ANOVA two way (3 Grupos $\mathrm{x} 2$ Testes) mostrou diferença significativa apenas no segundo fator $f(1,142)=18,031$ e $p<0,05$. A outra ANOVA realizada para o coeficiente de variação relativo (CVR) (3Grupos x 2Testes) mostrou uma diminuição significativa dessa medida do pré-teste para o pós-teste. De acordo com os resultados, o estabelecimento de metas não auxiliou na aprendizagem de habilidades esportivas. Os resultados foram discutidos em relação ao nível de habilidade dos sujeitos e às metas de processo e de produto.

Palavras-chave: Aprendizagem motora, estabelecimento de metas, basquetebol.

\section{ABSTRACT \\ Goal-setting effects on Basketball shot learning}

Goals can be divided into generic goals and specific goals. Generic goals belong to the group "do your best", while the specific goals are well defined and preferably quantitative. This strategy for goal-setting has been used with elite athletes, but little is known about its effects on motor skills learning. This question was investigated in a real teaching situation, during physical education classes, with children $(n=166)$ of the elementary school (11 years old), divided in three experimental groups: generic goals $(M G)$, specific goals $(M E)$ and without goals $(S M)$ in the task basketball shot. The ANOVA two way (3 groups X 2 tests) with repeated measures has just indicated a significant difference in the second factor $f(1,142)=18,031$ and $p<0,05$. Moreover, the another ANOVA conducted for the relative variation coefficient (CVR) (3 groups $x 2$ tests) showed that this measure went down significantly from the pre to the post test. According to the results, the goal setting didn't help the learning of sports skills. The results are discussed in relation to the subjects' skill level and the process and product goal setting.

Keywords: Motor learning, goal setting, basketball. 


\section{INTRODUÇÃO}

A estratégia de estabelecer metas para si próprio ou para uma coletividade pode ser vista como um traço característico do comportamento humano, observado na capacidade de projetar virtualmente condições futuras para guiar as suas ações presentes.

Particularmente nos últimos 25 anos, o uso de metas como uma técnica eficaz para o aumento do desempenho ou produtividade tem recebido uma abordagem teórica. Locke \& Latham (10), com base numa revisão de mais de 400 estudos realizados no contexto de desempenho industrial e organizacional, propõem que metas específicas e desafiadoras, na perspectiva de quem irá desempenhar a tarefa, provocam níveis mais altos de desempenho que metas fáceis ou metas gerais (do tipo "faça o melhor possível"). Uma meta, do ponto de vista psicológico, pode ser entendida como um compromisso com uma projeção de desempenho. $\mathrm{O}$ estabelecimento de meta pode ser visto, então, como uma estratégia motivacional, que busca dirigir e manter a atenção do executante para um determinado objetivo a ser alcançado, e assim, melhorar seu desempenho (9).

As metas a serem estabelecidas podem obedecer a critérios ou "atributos" diversos. Burton (2) destaca quatro: o grau de dificuldade da meta, a temporalidade da meta, a coletividade da meta e a especificidade da meta. O grau de dificuldade refere-se à distância hipotética entre o nível de desempenho atual do indivíduo e o nível de desempenho estabelecido como meta. Segundo Locke \& Latham (9), metas consideradas difíceis, porém atingíveis, produzem desempenho superior ao alcançado com o uso de metas fáceis. Entretanto, no campo esportivo, Kyllo \& Landers (8) mostram que as pesquisas sobre o estabelecimento de metas no campo esportivo não dão suporte a essa hipótese.

As metas também podem ser manipuladas temporalmente, isto é, as metas podem ser de longo prazo e de curso prazo. Para esse atributo, Locke \& Latham (10) apontam que o uso da meta de longo prazo combinada a metas de curto prazo levaria a um melhor desempenho, comparativamente ao uso de meta de longo prazo isoladamente. Entretanto, poucos estudos têm investigado essa hipótese.

Outro atributo é a "coletividade da tarefa", que se refere à possibilidade da meta ser estabelecida para o grupo ou para o indivíduo. Uma meta pode ser considerada coletiva ou de grupo quando estipulada para todo o grupo como uma unidade, e não a simples soma de metas individuais (20). Esses autores sugerem que o estabelecimento de metas coletivas deveria melhorar o desempenho de uma equipe, contudo, ressaltam que praticamente nenhum estudo foi conduzido para investigar a coletividade da meta e o desempenho de equipes esportivas.

Um último atributo refere-se à especificidade da meta ou grau de objetividade da meta. Com relação a esse atributo, dois tipos de metas têm sido investigados: meta genérica ("faça o melhor possível") e meta específica (por exemplo, "10 chutes corretos em 12 tentativas"). Locke \& Latham (10) predizem que metas difíceis e específicas produzem um efeito superior sobre o desempenho que metas genéricas. Segundo os autores, as metas específicas regulam melhor o comportamento em direção ao estado almejado, quando comparadas com metas vagas ou situações sem metas. O efeito da especificidade da meta tem sido alvo de vários estudos, entretanto, Weinberg (19) ressalta que apesar dos resultados positivos no campo da produção industrial e organizacional, no contexto esportivo, os resultados não têm sido conclusivos.

Nos últimos cinco anos, o tema estabelecimento de metas tem sido alvo de pesquisas em outras áreas relacionadas ao comportamento motor. Por exemplo, Ponte-Allan \& Giles (13), mostram que no processo de reabilitação de indivíduos acometidos por acidente vascular cerebral, os pacientes sem metas permaneceram mais tempo na unidade médica do que os pacientes para os quais havia sido estabelecida uma meta temporal para a recuperação das funções selecionadas. O efeito do estabelecimento de metas não tem recebido muita atenção por parte dos pesquisadores da área de aprendizagem motora. Uma das exceções é o trabalho de Cezar, Ugrinowitsch, Tani, Teixeira (3), em que se investigou o efeito de diferentes tipos de metas (específicas e genéricas) na aprendizagem do drible do basquetebol (teste da AAHPERD) (1). De acordo com os resultados, não houve diferença intergrupos, ou seja, não foi verificado o efeito do estabelecimento de metas na aprendizagem. Contudo, os sujeitos eram participantes de um programa de obesidade do Centro de Práticas Esportivas da Universidade de São Paulo (CEPEUSP), e foram divididos em dois grupos de acordo com critérios relacionados ao programa no qual estavam inscritos. Nesse programa, um grupo tinha orientação alimentar e participava de um programa de atividade física (grupo meta genérica - MG), e o outro somente participava da atividade física (grupo meta específica $\mathrm{ME}$ ). O grupo MG pode ter apresentado um bom 
desempenho no teste por causa do maior comprometimento com o programa de obesidade.

Freudenheim \& Tani (7) investigaram o efeito do estabelecimento de metas na aprendizagem em uma tarefa de flutuação no meio líquido. Foram utilizados três grupos experimentais: grupo meta genérica (MG), grupo meta específica (ME) e grupo meta específica combinada com metas de curto prazo (MC). Não houve diferenças intergrupos.

A escassez de trabalhos, assim como os resultados encontrados, não permitem ainda uma visão clara do papel do estabelecimento de metas na aprendizagem de habilidades motoras, especificamente nos estudos que tiveram o objetivo de testar a hipótese de Locke \& Latham (9). Assim, testar essa hipótese, com tarefas reais (por exemplo, aquelas utilizadas em aulas de Educação Física) pode ser visto como um importante desafio na área de Comportamento Motor $(15,18)$. Este estudo pretende investigar o efeito do estabelecimento de metas para a aprendizagem da habilidade arremesso do basquetebol, em uma situação real de ensino e aprendizagem, em aulas de Educação Física Escolar.

\section{MATERIAL E MÉTODO}

Participaram desse experimento 166 sujeitos de ambos os sexos, que cursavam a primeira série do ensino fundamental numa escola particular da zona Oeste da cidade de São Paulo, com idade variando entre 11 e 12 anos, cujos pais consentiram a participação na pesquisa. A tarefa consistiu em realizar o arremesso frontal a uma cesta oficial de basquetebol, à distância horizontal de dois metros da tabela oficial do jogo. A tarefa foi adaptada do teste da AAHPERD (1) (Figura 1).

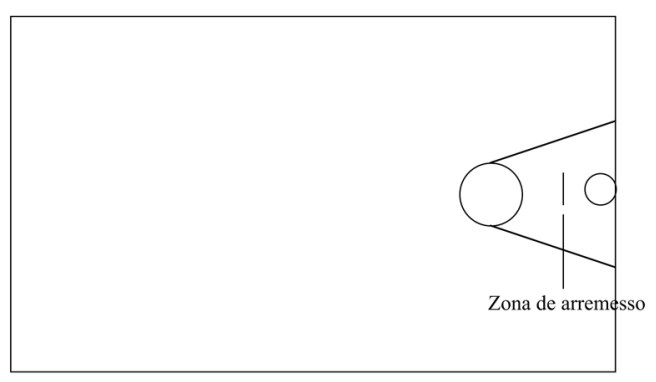

Figura 1 - Local do teste de arremesso do basquetebol adaptado de AAHPERD(1).
A amostra foi dividida em 3 grupos, de acordo com a classe a que pertenciam na escola. Isso foi feito com o objetivo de evitar a "contaminação" dos diferentes tipos de metas. O grupo meta genérica (MG) recebia a instrução "faça o maior número de cestas possível". O grupo meta específica (ME) recebia a instrução de qual o índice de acertos a ser alcançado ao final das nove sessões de prática. Esse número foi estipulado para cada aluno e era $20 \%$ superior ao número de cestas convertidos no pré-teste. O grupo sem meta (SM) recebia a instrução "faça 10 arremessos". Todos os sujeitos participavam normalmente das aulas de educação física; as instruções eram dadas no início de cada aula, seguido do momento dedicado à parte experimental, em que os sujeitos realizavam o mesmo número de arremessos do teste inicial (10 arremessos), totalizando 100 arremessos durante todo o experimento. Os alunos que faltaram em duas ou mais sessões de prática foram excluídos do estudo. Os sujeitos de cada grupo (MG, ME e SM) foram organizados em grupos de seis alunos (um dos grupos com sete integrantes) e cada um deles realizava um arremesso e seguia para o final da fila. Somente após todos realizarem o primeiro arremesso, era realizado o segundo, até que se totalizasse 10 arremessos. No caso de faltas (não comparecimento do aluno à aula), o grupo era composto pelo número de alunos presentes. Esse procedimento foi adotado em todas as sessões, incluindo o pré e pós-testes. Não foi fornecido feedback pedagógico sobre o resultado aos sujeitos, independentemente do grupo a que pertenciam, pois nessa tarefa ele era redundante com a informação visual.

Na primeira sessão, todos os sujeitos participaram de um pré-teste, que consistiu na realização de 10 arremessos a uma distância horizontal de dois metros da tabela oficial de basquetebol. Para cada arremesso convertido era computado um ponto, e para cada arremesso falho zero ponto. Seguiram-se oito sessões de prática, e na décima sessão foi realizado o pós-teste. As variáveis dependentes foram a média de pontos do teste (média de cestas convertidas) e a variabilidade nas execuções. Para a medida de variabilidade optou-se pelo coeficiente de variação (CVR) (6), uma vez que essa tem sido vista como a melhor medida para verificar a variabilidade (11). Com o objetivo de identificar as possíveis diferenças intra e intergrupo da média dos pontos alcançados no pré e pós-testes foi realizada uma ANOVA two-way (3 grupos $\mathrm{x} 2$ testes), com medidas repetidas no segundo fator. Esse procedimento auxilia a responder se os 
sujeitos aprenderam a habilidade, ou seja, tornaramse mais consistentes, e também qual tipo de tratamento influenciou mais a aprendizagem, sendo estabelecido o $\mathrm{p}<0,05$. O mesmo teste estatístico foi aplicado sobre o coeficiente de variação relativa.

\section{RESULTADOS}

Apesar da análise descritiva indicar que os grupos MG e ME apresentaram uma melhora mais acentuada no desempenho em relação ao grupo SM (Figura 2), a ANOVA two way com medidas repetidas indicou diferença significativa somente no segundo fator (testes), com $\mathrm{f}(1,142)=18,031$ e $\mathrm{p}<0,01$, não havendo diferença intergrupos e interação entre os dois fatores para $\mathrm{p}<0,05$.

De acordo com os resultados, o grupo ME apresentou desempenho superior ao grupo MG, e ambos superiores ao SM. Contudo, esse experimento não corroborou a hipótese de que o estabelecimento de metas específicas auxilia a aprendizagem, como propõem Locke \& Latham (9) e Ponte-Allan \& Giles (13), pois não houve diferença estatisticamente significativa intergrupos.

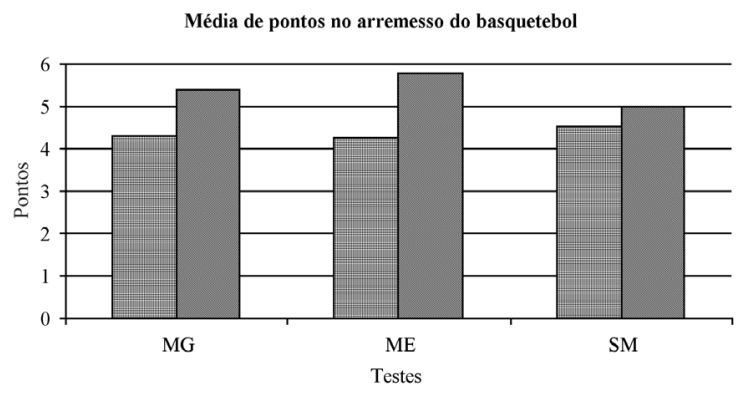

Figura 2 - Média de arremessos certos no pré e pós-testes em função do grupo ( $M G, M E$, SM).

Os três grupos tiveram alteração na variabilidade (Figura 3). A análise do CVR desse experimento indicou a diminuição da variabilidade do pré para o pós-teste nos três grupos. A ANOVA two way (3 grupos $\mathrm{x} 2$ testes) com medidas repetidas indicou diferença significativa no segundo fator (testes), com $\mathrm{f}(1,142)=18,031$ e $\mathrm{p}<0,05$, e não houve diferença no primeiro fator e nem interação entre os dois fatores para $\mathrm{p}<0,05$.
Com base nesses resultados, pode-se inferir que os três grupos apresentaram uma melhora na média de pontos e diminuíram a variabilidade do erro do início para o final do experimento, tornando-se mais consistentes. Entretanto, apesar do aumento da consistência e diminuição da variabilidade no desempenho do arremesso do basquetebol nos três grupos, não houve efeito do estabelecimento de metas na aprendizagem.

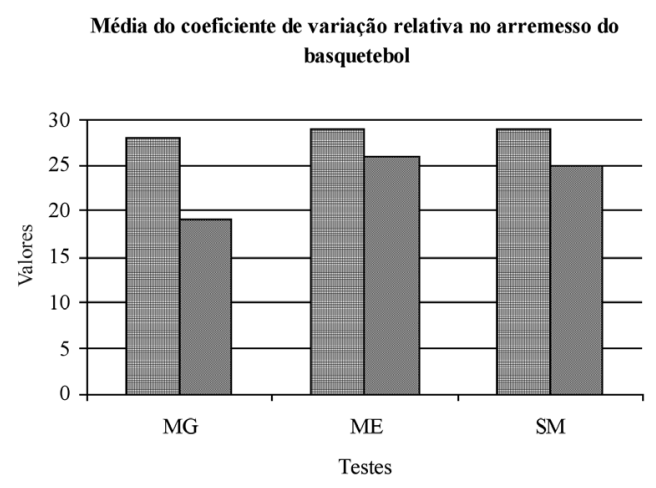

Figura 3 - Coeficiente de variação relativa dos grupos $M G, M \varepsilon$ e SM no pré e pós-testes.

\section{DISCUSSÃO}

Os resultados dos testes estatísticos não corroboraram a hipótese de que as metas específicas facilitam a aquisição de habilidades motoras (9), pois os três grupos apresentaram melhoras no desempenho do início para o final do período de prática.

Uma questão levantada após se observar a igualdade entre os grupos ME e MG é que os sujeitos dos grupos MG e SM podem ter estabelecido metas específicas individualmente, o que eliminaria a diferença entre os grupos. Entretanto, segundo comunicação pessoal do professor de educação física que conduziu as sessões experimentais, isso parece não ter ocorrido. O que ocorreu, segundo o mesmo, foi a competição entre os sujeitos, mas esse foi um fenômeno que ocorreu igualmente em todos os grupos. Entretanto, uma diminuição no controle é comum em pesquisas que buscam testar predições teóricas básicas em situações reais $(4,15,18)$. 
Apesar de não termos detectado diferenças estatisticamente significantes entre os grupos, observamos que houve uma tendência de melhor desempenho do grupo ME em relação aos demais. A mesma tendência foi observada em relação à medida de variabilidade (CVR), pois o grupo MG apresentou uma tendência a menor variabilidade quando comparado aos grupos ME e SM. Vale ressaltar que, em ambos os casos, o grupo SM teve desempenho inferior aos outros dois grupos, o que pode ser um indicativo de que o estabelecimento de metas, sejam genéricas, sejam específicas, favorece mais a aprendizagem do que a ausência de metas.

Um outro fator a ser considerado é que a medida de aprendizagem utilizada pode não ser adequada.

Parece ser necessário utilizar um instrumento que seja mais sensível e que discrimine melhor outros aspectos da aprendizagem (padrão de movimento e de controle de parâmetros). Pelos alunos serem iniciantes, e portanto, estarem voltados para a formação de uma idéia geral do movimento, os efeitos do processo de aprendizagem podem ser mais nítidos no padrão de execução do movimento. Ainda em relação às metas específicas, elas podem ser de longo prazo, como nesse estudo, ou de longo prazo combinadas com metas de curto prazo (9). As metas de curto prazo parecem direcionar mais a atenção dos sujeitos do que metas de longo prazo $(7,16)$, pois as metas de longo prazo freqüentemente são muito distantes para motivar ações imediatas (8).

Particularmente com crianças, esse parece ser mais um caminho a percorrer para esclarecer o efeito do estabelecimento de metas na aprendizagem de habilidades motoras.

Também é necessário ressaltar que, nesse estudo, os sujeitos não foram separados pelo tipo de orientação que possuíam (à tarefa ou ao ego) $(5,12,14,17)$. Talvez seja importante verificar qual a fase de aprendizagem que o sujeito se encontra, para então propor diferentes tipos de metas. Por exemplo, um sujeito que está no estágio inicial de aprendizagem deve ter a atenção voltada para a aprendizagem do processo de organização de estratégias para atingir o objetivo. Nesse momento, metas de processo devem auxiliar a manter a atenção nessas estratégias. Já um atleta que apresenta um alto grau de domínio da habilidade deveria utilizar, pelo menos, metas de resultado, pois uma competição está diretamente relacionada a vencer oponentes. Como os sujeitos que participaram desse estudo não dominavam as habilidades praticadas, seria importante utilizar metas de processo, e não de desempenho.

Entretanto os autores desconhecem formas de operacionalizar metas de processo, como por exemplo, estipular índices de dificuldade em metas qualitativas, ou mesmo propor medidas operacionais robustas para avaliação qualitativa do desempenho. Os crescentes avanços na área de cinemática do movimento humano têm se mostrado promissores quanto ao segundo problema, entretanto, não foi encontrado na literatura uma resposta satisfatória para o primeiro problema.

\section{CONCLUSÕES}

Apesar do grupo ME ter uma tendência de melhor desempenho que os grupos MG e SM, esse resultado não foi confirmado pela análise estatística, o mesmo ocorrendo em relação à diminuição da variabilidade. São necessários novos estudos que investiguem o estabelecimento de metas na aprendizagem em outras tarefas. Entretanto, uma limitação que precisa ser superada em futuros estudos, para que avancemos na compreensão desse fenômeno, refere-se à manipulação de metas de processo.

\section{CORRESPONDÊNCIA}

\section{Herbert Ugrinowitsch}

Laboratório de Comportamento Motor (LACOM)

Escola de Educação Física e Esportes

Universidade de São Paulo

Avenida Prof. Mello Moraes, 65

Caixa Postal 5349

05508 - 900 Butantã

São Paulo, SP

Brasil

nowitsch@usp.br 


\section{REFERÊNCIAS BIBLIOGRÁFICAS}

1. American Association for Health, Physical Education and Recreation. (1969). Skill test manual: basketball for boys and girls. Washington: AAHPERD.

2. Burton, D. (1994). Goal setting in sport. In: Singer, R.N. \& Tennant, L.R. (Eds.). Handbook of Research of Sport Psychology. New York: MacNillan, 467-491.

3. Cezar, C.; Ugrinowitsch, H.; Tani, G.; Teixeira, L.R. (1997). Estabelecimento de metas. In: Anais do V Simpósio Paulista de Educação Física, 45.

4. Christina, R. (1989). Motor learning: future lines of research. In: Safrit, M.J.; Eckert, H.M. (Eds.). The cutting edge in physical education and exercise science research. (American Academy of Physical Education Papers, 20). Champaign, Ill.: Human Kinetics, 26-41.

5. Duda, J.L. (1996). Maximizing motivation in sport and physical education among children and adolescents: The case for greater task involvement. Quest, 48: 290-302.

6. Eisenhauer, J.G. (1993). A measure of relative dispersion. Teaching Statistics, 2(14): 37-39.

7. Freudenheim, A.; Tani, G. (1998). O efeito do estabelecimento de metas na aquisição de uma habilidade de sustentação no meio líquido em crianças. Revista da APEF, 1(13): 05-11.

8. Kyllo, L.B.; Landers, D.M. (1995). Goal setting in sport and exercise: A research synthesis to resolve the controversy. J. of Sport \& Exercise Psychol, 17: 117-137.

9. Locke, E.A.; Latham, G.P. (1985). The application of goal setting to sports. J. of Sport Psychol., 7: 205-222.

10. Locke, E.A.; Latham, G.P. (1990). A theory of goal setting and task motivation. Englewood Cliffs, NJ: Prentice Hall.

11. Newell, K.M.(1996). Change in Movement and Skill: Learning, Retention and Transfer. In: Latash, M.L.; Turvey, M.T. Dexterety and its Development with on Dexterity and its Development by N.A. Bernstein. New Jersey: Mahwah.

12. Ntoumanis, N.; Biddle, S. (1998). The relationship between competitive anxiety, achievement goals, and motivational climates. Research Quarterly for Exercise and Sport, 2(69):176-187.

13. Ponte-Allan, M.; Giles, G.M. (1998). Goal setting and functional outcomes in rehabilitation. The American J. of Occupational Therapy, 6(53): 646-649.

14. Sarrazin, P.; Famose, J.P. (1999). Children's goal and motivation in physical education. In: Auweele, Y.V.; Bakker, F.; Biddle, S.; Durand, M.; Seiler, R. (Eds). Psychology for Physical Educators. Champaign, Illinois: Human Kinetics. 27-50.

15. Tani, G. (1992). Contribuições da aprendizagem motora à Educação Física: uma análise crítica. Revista Paulista de Educação Física, 2(6): 65-72.

16. Tenenbaum, G.; Bar-Eli, M.; Yaaron, M. (1999). The dynamics of goal-setting: Interactive effects of goal difficulty, goal specificity and duration of practice time intervals. Int J. of Sport Psychol., 30:325-338.

17. Treasure, D.C.; Roberts, G.C. (1998). Relationship between female adolescents' achievement goal orientations, perceptions of the motivational climate, belief about success and sources of satisfaction in basketball. Int. J. Sport Psychol., 29:211-230.

18. Ugrinowitsch, H. (1999). Pesquisa de síntese em Biodinâmica do movimento. In: Anais do II Simpósio de PósGraduação da Escola de Educação Física e Esportes da Universidade São Paulo. São Paulo: USP, 68.
19. Weinberg, R.S. (1992). Goal setting and motor performance: a review and critique. In: Roberts, G.C. (Ed.) Motivation in sport and exercise. Champaign, Illinois: Human Kinetics. 177-197.

20. Widmeyer, W.N.; Ducharme, K. (1997). Team building through team goal setting. J. of Applied Sport Psychol., 9: 97-113. 